SNUTP 94-14

February 1994

\title{
Schrödinger Fields on the Plane with non-Abelian Chern-Simons Interactions
}

\author{
Won Tae Kim* and Choonkyu Lee \\ Center for Theoretical Physics and Department of Physics, \\ Seoul National University, Seoul 151-742, Korea
}

\begin{abstract}
Physical content of the nonrelativistic quantum field theory with non-Abelian Chern-Simons interactions is clarified with the help of the equivalent first- quantized description which we derive in any physical gauge.
\end{abstract}

(Submitted to: Physical Review D)

*E-mail address: wtkim@phya.snu.ac.kr 


\section{INTRODUCTION}

Quantal structure of the nonrelativistic field theory on the plane with Abelian Chern-Simons interactions has been discussed recently in Ref. [1] : it reduces to the theory of particles obeying exotic Abelian statistics, usually called anyons [2]. In two spatial dimensions, it is known that particles obeying non-Abelian braid statistics are also possible [3]. The latter case is realized if particles are allowed to couple to statistical gauge fields whose dynamics is governed by the non-Abelian Chern-Simons Lagrange density [4]

$$
\mathcal{L}_{C S}=-\kappa \epsilon^{\mu \nu \lambda} \operatorname{tr}\left(A_{\mu} \partial_{\nu} A_{\lambda}+\frac{2}{3} A_{\mu} A_{\nu} A_{\lambda}\right)
$$

where $\kappa$ is a dimensionless constant and $A_{\mu}=A_{\mu}^{a} T^{a}$ is the matrix-valed gauge connection. (The $T^{a}$ 's are antihermitian group generators satisfying $\left[T^{a}, T^{b}\right]=f^{a b c} T^{c}$

and $\left.\operatorname{tr}\left(T^{a} T^{b}\right)=-\frac{1}{2} \delta^{a b}\right)$. Gauge invariance of the theory requires quantization of the parameter $\kappa, \kappa=\frac{n}{4 \pi}(n \in Z)[5]$.

Quantum mechanical or first-quantized description of these non-Abelian ChernSimons particles has been given in the so-called holomorphic gauge [6, 7]. In more usual (e.g. Coulomb) gauges, the nonlinear nature of the Gauss law has been a major obstacle to the explicit treatment. As an alternative formulation, one can also contemplate the field-theoretic or second-quantized description of these particles, i.e. the nonAbelian extension of the treatment given in Ref. [1]. In this paper, we present such a treatment and show explicitly that this system has an equivalent first-quantized description with all the desired properties. Note that, when one posits the secondquantized Hamiltonian, the holomorphic gauge choice becomes problematic since the corresponding Hamiltonian possesses no obvious Hermiticity property. But for our purpose at least, any gauge choice preserving the Hermiticity of the vector potentials $A_{i}^{a}$ will do. In the next section, we will specify our field theory system and then derive the many-body Schrödinger equation implied by it. The last section is devoted to the discussion of our finding. 


\section{NONRELATIVISTIC FIELD THEORY AND ITS FIRST-QUANTIZED DESCRIPTION}

The second-quantized description utilizes the (complex) Schrödinger fields $\psi_{\alpha}(\mathbf{r}, t)$ which interact minimally with non-Abelian Chern-Simons gauge fields $A_{\mu}^{a}$. Here, $\mathbf{r}=(x, y)$ and $\alpha$ denotes the internal index associated with a specific representation of the gauge group appropriate to given particles. We work with Heisenberg-picture field operators. Then, based on the standard Lagrangian density which includes $\mathcal{L}_{c s}$ given above, we readily obtain the Hamiltonian of the system (we set $\hbar=1$ and $i=1,2$ ),

$$
\begin{aligned}
H & =\frac{1}{2 m} \int d^{2} \mathbf{r} \psi^{\dagger}(\mathbf{r}, t)\left(\overleftarrow{\partial_{i}}-A_{i}^{a}(\mathbf{r}, t) T^{a}\right)\left(\partial_{i}+A_{i}^{b}(\mathbf{r}, t) T^{b}\right) \psi(\mathbf{r}, t) \\
& \equiv \frac{1}{2 m} \int d^{2} \mathbf{r}\left(D_{i} \psi\right)^{\dagger}(\mathbf{r}, t)\left(D_{i} \psi\right)(\mathbf{r}, t)
\end{aligned}
$$

together with the Gauss laws which relate the operators $A_{i}^{a}(\mathbf{r}, t)$ to non-Abelian matter densities $\rho^{a}(\mathbf{r}, t)=i \psi^{\dagger}(\mathbf{r}, t) T^{a} \psi(\mathbf{r}, t)$ :

$$
\frac{1}{2} \epsilon^{i j} F_{i j}^{a} \equiv \epsilon^{i j} \partial_{i} A_{j}^{a}+\frac{1}{2} \epsilon^{i j} f^{a b c} A_{i}^{b} A_{j}^{c}=-\frac{1}{\kappa} \rho^{a}
$$

If one wishes, one may also include in the Hamiltonian additional gauge-invariant interactions involving matter fields only. The Schrödinger field operators $\psi_{\alpha}$ and $\psi_{\alpha}^{\dagger}$ satisfy the usual equal-time commutation relations

$$
\begin{aligned}
{\left[\psi_{\alpha}(\mathbf{r}, t), \psi_{\beta}\left(\mathbf{r}^{\prime}, t\right)\right] } & =\left[\psi_{\alpha}^{\dagger}(\mathbf{r}, t), \psi_{\beta}^{\dagger}\left(\mathbf{r}^{\prime}, t\right)\right]=0 \\
{\left[\psi_{\alpha}(\mathbf{r}, t), \psi_{\beta}^{\dagger}\left(\mathbf{r}^{\prime}, t\right)\right] } & =\delta_{\alpha \beta} \delta^{2}\left(\mathbf{r}-\mathbf{r}^{\prime}\right)
\end{aligned}
$$

(We take a bosonic algebra for definiteness; similar analysis can be given with a fermionic algebra.) A simple consequence of these commutation relations is: $\left[\rho^{a}(\mathbf{r}, t)\right.$, $\left.\rho^{b}\left(\mathbf{r}^{\prime}, t\right)\right]=i f^{a b c} \delta^{2}\left(\mathbf{r}-\mathbf{r}^{\prime}\right) \rho^{c}(\mathbf{r}, t)$. We have now specified our system completely.

Does this nonrelativistic quantum field theory describe the same physical system as that discussed in Refs. [6, 7] ? This can be answered by deducing the equivalent first-quantized description. What makes the problem nontrivial (say, compared to the Abelian case [1, 8]) is that, in the present case, the operators $A_{i}^{a}(\mathbf{r}, t)$ are specified 
only implicitly through Eq. (3). We also need a properly ordered second-quantized Hamiltonian. It turns out that the 'correct' form of the Hamiltonian operator is given by our expression (2), with various operators ordered precisely in the form written there. This gives the correct (Hermitian) Hamiltonian in the sense that the most natural first-quantized description then follows.

The source of difficulty with Eq. (3) is of course the nonlinear term in the nonAbelian field strength $F_{i j}^{a}$. (This nonlinear term would drop out in the axial gauge $\left(A_{1}^{a} \equiv 0\right)$, but it is not so in more safe gauges where the fields $A_{i}^{a}$ may be assumed to vanish at spatial infinity). Still it should always be possible to represent the solution $A_{i}^{a}(\mathbf{r}, t)$ by the ordered form

$$
\begin{aligned}
A_{i}^{a}(\mathbf{r}, t)=W_{i}^{(0) a}(\mathbf{r})+ & \sum_{\substack{n=1\\
}}^{\infty} \int d^{2} \mathbf{r}_{1} \cdots d^{2} \mathbf{r}_{n} \psi^{\dagger}\left(\mathbf{r}_{1}, t\right) \cdots \psi^{\dagger}\left(\mathbf{r}_{n}, t\right) W_{i}^{(n) a}\left(\mathbf{r} ; \mathbf{r}_{1}, T_{(1)}, \cdots, \mathbf{r}_{n}, T_{(n)}\right) \\
& \psi\left(\mathbf{r}_{1}, t\right) \cdots \psi\left(\mathbf{r}_{n}, t\right)
\end{aligned}
$$

with suitable (matrix-valued) c-number functions $W_{i}^{(n) a}$ satisfying the symmetry restriction

$$
W_{i}^{(n) a}\left(\mathbf{r} ; \cdots, \mathbf{r}_{p}, T_{(p)}, \cdots, \mathbf{r}_{q}, T_{(q)}, \cdots\right)=W_{i}^{(n) a}\left(\mathbf{r} ; \cdots, \mathbf{r}_{q}, T_{(q)}, \cdots, \mathbf{r}_{p}, T_{(p)}, \cdots\right)
$$

In Eq. (5), the $T_{(p)}$ in $W_{i}^{(n) a}$ denote generator matrices acting on $\psi\left(\mathbf{r}_{p}, t\right)$ (and on $\psi^{\dagger}\left(\mathbf{r}_{p}, t\right)$ from the right) and so satisfy $\left[T_{(p)}^{a}, T_{(q)}^{b}\right]=\delta_{p q} f^{a b c} T_{(p)}^{c}$. By inserting the form (5) into Eq. (3), we then obtain the conditions that the functions $W_{i}^{(n) a}$ should satisfy. For the c-number contribution $W_{i}^{(0) a}(\mathbf{r})$, this consideration immediately tells us that it is necessarily a pure gauge; we will set $W_{i}^{(0) a}(\mathbf{r}) \equiv 0$ hereafter, as a part of our gauge choice. Now the rest of the functions $W_{i}^{(n) a}\left(\mathbf{r} ; \mathbf{r}_{1}, T_{(1)}, \cdots, \mathbf{r}_{n}, T_{(n))}\right.$, or $W_{i}^{(n) a}(\mathbf{r} ; 1,2, \cdots, n)$ in short, are required to solve the following equations iteratively:

$$
\begin{aligned}
& \epsilon^{i j} \partial_{i} W_{j}^{(1) a}(\mathbf{r} ; 1)+\frac{1}{2} \epsilon^{i j} f^{a b c} W_{i}^{(1) b}(\mathbf{r} ; 1) W_{j}^{(1) c}(\mathbf{r} ; 1)=-\frac{i}{\kappa} T_{(1)}^{a} \delta\left(\mathbf{r}-\mathbf{r}_{1}\right), \\
& \epsilon^{i j} \partial_{i} W_{j}^{(2) a}(\mathbf{r} ; 1,2)+\frac{1}{2} \epsilon^{i j} f^{a b c}\left\{W_{i}^{(1) b}(\mathbf{r} ; 1) W_{j}^{(1) c}(\mathbf{r} ; 2)+\left(W_{i}^{(1) b}(\mathbf{r} ; 1)+W_{i}^{(1) b}(\mathbf{r} ; 2)\right) W_{j}^{(2) c}(\mathbf{r} ; 1,2)\right. \\
& \left.+W_{i}^{(2) b}(\mathbf{r} ; 1,2)\left(W_{j}^{(1) c}(\mathbf{r} ; 1)+W_{j}^{(1) c}(\mathbf{r} ; 2)\right)+2 W_{i}^{(2) b}(\mathbf{r} ; 1,2) W_{j}^{(2) c}(\mathbf{r} ; 1,2)\right\}=0, \\
& \epsilon^{i j} \partial_{i} W_{j}^{(3) a}(\mathbf{r} ; 1,2,3)+\frac{1}{2} \epsilon^{i j} f^{a b c}\left\{W_{i}^{(1) b}(\mathbf{r} ; 1) W_{j}^{(2) b}(\mathbf{r} ; 2,3)+\cdots\right\}=0, \\
& \cdots \text {, etc. }
\end{aligned}
$$


Any particular set of solutions $W_{i}^{(n) a}$ satisfying these equations may be chosen for our subsequent developments.

While the first of Eq. (7) has a simple solution (in the Coulomb gauge),

$$
W_{i}^{(1) a}(\mathbf{r} ; 1)=\frac{i}{\kappa} \epsilon^{i j} \partial_{j} G\left(\mathbf{r}-\mathbf{r}_{1}\right) T_{(1)}^{a}, \quad\left(G\left(\mathbf{r}-\mathbf{r}_{1}\right)=\frac{1}{2 \pi} \ln \left|\mathbf{r}-\mathbf{r}_{1}\right|\right)
$$

it is a nontrivial task to solve the rest of Eq. (7) and thereby make available explicit forms for $W_{i}^{(n) a}$ with $n \geq 2$. (Note that if one chose the axial gauge, all $W_{i}^{(n) a}$ with $n \neq 1$ could be taken to be zero; but, the expression for $W_{i}^{(1) a}\left(\mathbf{r} ; \mathbf{r}_{1}, T_{(1)}\right)$ assumes a very awkward form). Despite the lack of explicit knowledge on $W_{i}^{(n) a}$ for $n \geq 2$, the following characterization of the functions $W_{i}^{(n) a}$ will prove useful later. For arbitrary $N(\geq 2)$, suppose we form the c-number vector potential,

$$
\begin{aligned}
\mathcal{A}_{i}^{a}(\mathbf{r} ; 1, \cdots, N-1) & =\sum_{p=1}^{N-1} W_{i}^{(1) a}(\mathbf{r} ; p)+\sum_{p} \sum_{q \neq p} W_{i}^{(2) a}(\mathbf{r} ; p, q)+\sum_{p} \sum_{q \neq p} \sum_{r \neq p, q} W_{i}^{(3) a}(\mathbf{r} ; p, q, r) \\
& +\cdots+\sum_{p} \sum_{q \neq p} \cdots \sum_{s \neq p, q, \cdots, s} W_{i}^{(N-1) a}(\mathbf{r} ; p, q, \cdots, s)
\end{aligned}
$$

then, as can readily be verified using Eq. (7), this provides the solution to the equations

$$
\frac{1}{2} \epsilon^{i j}\left(\partial_{i} \mathcal{A}_{j}^{a}-\partial_{j} \mathcal{A}_{i}^{a}+f^{a b c} \mathcal{A}_{i}^{b} \mathcal{A}_{j}^{c}\right)=-\frac{i}{\kappa} \sum_{p=1}^{N-1} T_{(p)}^{a} \delta^{2}\left(\mathbf{r}-\mathbf{r}_{p}\right) .
$$

So this potential defines a flat connection except at some discrete points; $\mathcal{A}_{i}^{a}(\mathbf{r} ; 1, \cdots, N-$ 1) describes the classical non-Abelian Chern-Simons field configuration when $N-1$ nonAbelian point sources are situated at positions $\mathbf{r}=\mathbf{r}_{p} \quad(p=1, \cdots, N-1)$. In fact one may completely specify the functions $W_{i}^{(n) a}$ through Eqs. (9), (10) and (6). Also it will soon become evident that if one is concerned with the $N$-particle sector of the given system, one is in fact allowed to truncate the above series expression (Eq. (5)) for the vector potentials $A_{i}^{a}(\mathbf{r}, t)$ to $n=N-1$, all terms after $n=N-1$ in the sum not contributing at all.

The complicated expression obtained by inserting the form (5) into Eq. (2) is our Hamiltonian. The corresponding Heisenberg equations of motion are

$$
i \frac{\partial \psi_{\alpha}(\mathbf{r}, t)}{\partial t}=\left[\psi_{\alpha}(\mathbf{r}, t), H\right]
$$




$$
\begin{aligned}
= & -\frac{1}{2 m}\left(\vec{D}^{2} \psi\right)_{\alpha}(\mathbf{r}, t) \\
& +\frac{1}{2 m} \int d^{2} \mathbf{r}^{\prime} \psi^{\dagger}\left(\mathbf{r}^{\prime}, t\right)\left\{\left(\overleftarrow{\partial}_{i}^{\prime}-A_{i}^{a}\left(\mathbf{r}^{\prime}, t\right) T^{\prime a}\right) K_{i}^{b}\left(\mathbf{r}^{\prime}, \mathbf{r}, t\right)_{\alpha \beta} T^{\prime b}\right. \\
& \left.\quad-K_{i}^{b}\left(\mathbf{r}^{\prime}, \mathbf{r}, t\right)_{\alpha \beta} T^{\prime b}\left(\partial_{i}^{\prime}+A_{i}^{a}\left(\mathbf{r}^{\prime}, t\right) T^{\prime a}\right)\right\} \psi\left(\mathbf{r}^{\prime}, t\right) \psi_{\beta}(\mathbf{r}, t) \\
& -\frac{1}{2 m} \int d^{2} \mathbf{r}^{\prime} \psi^{\dagger}\left(\mathbf{r}^{\prime}, t\right) K_{i}^{a}\left(\mathbf{r}^{\prime}, \mathbf{r}, t\right)_{\alpha \beta} T^{\prime a} K_{i}^{b}\left(\mathbf{r}^{\prime}, \mathbf{r}, t\right)_{\beta \gamma} T^{\prime b} \psi\left(\mathbf{r}^{\prime}, t\right) \psi_{\gamma}(\mathbf{r}, t)
\end{aligned}
$$

where the generator matrices $T^{\prime a}$ act only on the fields with coordinate $\mathbf{r}^{\prime}$, and we have defined the operators $K_{i}^{a}\left(\mathbf{r}^{\prime}, \mathbf{r}, t\right)_{\alpha \beta}$ by the relation

$$
\begin{aligned}
{\left[\psi_{\alpha}\left(\mathbf{r}_{1}, t\right), A_{i}^{a}\left(\mathbf{r}^{\prime}, t\right)\right]=} & \left\{\sum_{n=1}^{\infty} \int d^{2} \mathbf{r}_{2} \cdots d^{2} \mathbf{r}_{n} \psi^{\dagger}\left(\mathbf{r}_{2}, t\right) n W_{i}^{(n) a}\left(\mathbf{r}^{\prime} ; 1,2, \cdots, n\right)_{\alpha \beta}\right. \\
\left.\cdot \psi\left(\mathbf{r}_{2}, t\right) \cdots \psi\left(\mathbf{r}_{n}, t\right)\right\} \psi_{\beta}\left(\mathbf{r}_{1}, t\right) & \\
\equiv & K_{i}^{a}\left(\mathbf{r}^{\prime}, \mathbf{r}_{1}, t\right)_{\alpha \beta} \psi_{\beta}\left(\mathbf{r}_{1}, t\right)
\end{aligned}
$$

Here the indeces $\alpha, \beta$ are used in association with the matrices $T_{(1)}$. The last term in the right hand side of Eq. (11) corresponds to the quantum operator ordering correction (an analogous term appears in the Abelian case as well [1]); this ordering term is important to obain the correct first-quantized description below.

Now let $|\Phi\rangle$ denote any Heisenberg-picture $N$-particle state vector. Then the corresponding Schrödinger wave function is given by

$$
\Phi\left(\mathbf{r}_{1}, \alpha_{1}, \cdots, \mathbf{r}_{N}, \alpha_{N} ; t\right)=\left\langle 0\left|\frac{1}{\sqrt{N !}} \psi_{\alpha_{1}}\left(\mathbf{r}_{1}, t\right) \cdots \psi_{\alpha_{N}}\left(\mathbf{r}_{N}, t\right)\right| \Phi\right\rangle
$$

where the nonrelativistic vacuum $|0\rangle$ satisfies the condition $\psi_{\alpha}(\mathbf{r}, t)|0\rangle=0$. Thanks to the operator field equations (11), we may express the time derivative of this $N$-body wave function as

$$
i \frac{\partial}{\partial t} \Phi\left(\mathbf{r}_{1}, \alpha_{1}, \cdots, \mathbf{r}_{N}, \alpha_{N} ; t\right)=A+B+C+D
$$

with

$$
\begin{aligned}
A= & \sum_{p=1}^{N}\langle 0| \frac{1}{\sqrt{N !}} \psi_{\alpha_{1}}\left(\mathbf{r}_{1}, t\right) \cdots \psi_{\alpha_{p-1}}\left(\mathbf{r}_{p-1}, t\right)\left\{-\frac{1}{2 m}\left(D_{i} D_{i} \psi\right)_{\alpha_{p}}\left(\mathbf{r}_{p}, t\right)\right\} \\
& \cdot \psi_{\alpha_{p+1}}\left(\mathbf{r}_{p+1}, t\right) \cdots \psi_{\alpha_{N}}\left(\mathbf{r}_{N}, t\right)|\Phi\rangle
\end{aligned}
$$




$$
\begin{aligned}
B= & \sum_{p=1}^{N}\langle 0| \frac{1}{\sqrt{N !}} \psi_{\alpha_{1}}\left(\mathbf{r}_{1}, t\right) \cdots \psi_{\alpha_{p-1}}\left(\mathbf{r}_{p-1}, t\right) \\
& \cdot\left\{\frac{1}{2 m} \int d^{2} \mathbf{r}^{\prime} \psi^{\dagger}\left(\mathbf{r}^{\prime}, t\right)\left(\overleftarrow{\partial}_{i}{ }^{\prime}-A_{i}^{a}\left(\mathbf{r}^{\prime}, t\right) T^{\prime a}\right) K_{i}^{b}\left(\mathbf{r}^{\prime}, \mathbf{r}_{p}, t\right)_{\alpha_{p} \beta_{p}} T^{\prime b} \psi\left(\mathbf{r}^{\prime}, t\right) \psi_{\beta}\left(\mathbf{r}_{p}, t\right)\right\} \\
& \cdot \psi_{\alpha_{p+1}}\left(\mathbf{r}_{p+1}, t\right) \cdots \psi_{\alpha_{N}}\left(\mathbf{r}_{N}, t\right)|\Phi\rangle \\
C= & \sum_{p=1}^{N}\langle 0| \frac{1}{\sqrt{N !}} \psi_{\alpha_{1}}\left(\mathbf{r}_{1}, t\right) \cdots \psi_{\alpha_{p-1}}\left(\mathbf{r}_{p-1}, t\right) \\
& \cdot\left\{-\frac{1}{2 m} \int d^{2} \mathbf{r}^{\prime} \psi^{\dagger}\left(\mathbf{r}^{\prime}, t\right) K_{i}^{b}\left(\mathbf{r}^{\prime}, \mathbf{r}_{p}, t\right)_{\alpha_{p} \beta_{p}} T^{\prime b}\left(\partial_{i}^{\prime}+A_{i}^{a}\left(\mathbf{r}^{\prime}, t\right) T^{a}\right) \psi\left(\mathbf{r}^{\prime}, t\right) \psi_{\beta_{p}}\left(\mathbf{r}_{p}, t\right)\right\} \\
& \cdot \psi_{\alpha_{p+1}}\left(\mathbf{r}_{p+1}, t\right) \cdots \psi_{\alpha_{N}}\left(\mathbf{r}_{N}, t\right)|\Phi\rangle, \\
D= & \sum_{p=1}^{N}\langle 0| \frac{1}{\sqrt{N !}} \psi_{\alpha_{1}}\left(\mathbf{r}_{1}, t\right) \cdots \psi_{\alpha_{p-1}}\left(\mathbf{r}_{p-1}, t\right) \\
& \cdot\left\{-\frac{1}{2 m} \int d^{2} \mathbf{r}^{\prime} \psi^{\dagger}\left(\mathbf{r}^{\prime}, t\right) K_{i}^{b}\left(\mathbf{r}^{\prime}, \mathbf{r}_{p}, t\right)_{\alpha_{p} \beta_{p}} T^{\prime b} K_{i}^{a}\left(\mathbf{r}^{\prime}, \mathbf{r}_{p}, t\right)_{\beta_{p} \gamma_{p}} T^{\prime a} \psi\left(\mathbf{r}^{\prime}, t\right) \psi_{\gamma_{p}}\left(\mathbf{r}_{p}, t\right)\right\} \\
& \cdot \psi_{\alpha_{p+1}}\left(\mathbf{r}_{p+1}, t\right) \cdots \psi_{\alpha_{N}}\left(\mathbf{r}_{N}, t\right)|\Phi\rangle
\end{aligned}
$$

The matrix elements given here may be calculated by considering the procedure of relocating the operators $\psi_{\alpha_{1}}\left(\mathbf{r}_{1}, t\right) \cdots \psi_{\alpha_{p-1}}\left(\mathbf{r}_{p-1}, t\right)$, one by one, to the right of those inside the curly brackets, while taking into account various commutator terms generated in effecting the relocation. Then all that has to be done is to collect those commutator terms ( here remember that $\langle 0| \psi_{\alpha}^{\dagger}(\mathbf{r}, t)=0$ ) and evaluate them using the relations like that in Eq. (12). For more on this procedure, readers may consult Ref. [8]. The result of this manipulation is

$$
\begin{aligned}
A=-\frac{1}{2 m} \sum_{p=1}^{N}\left\{\partial_{i}^{(p)}+\left(\sum_{q_{1}(<p)} W_{i}^{(1) a}\left(\mathbf{r}_{p} ; q_{1}\right)+\sum_{q_{1}(<p)} \sum_{q_{2}(<p) \neq q_{1}} W_{i}^{(2) a}\left(\mathbf{r}_{p} ; q_{1}, q_{2}\right)+\cdots\right.\right. \\
\left.\left.+\sum_{q_{1}(<p)} \ldots \sum_{q_{p-1}(<p) \neq q_{1}, \cdots, q_{p-2}} W_{i}^{(p-1) a}\left(\mathbf{r}_{p} ; q_{1}, \cdots, q_{p-1}\right)\right) T_{(p)}^{a}\right\} \\
2 \\
B=-\frac{1}{2 m} \sum_{p=1}^{N} \sum_{q_{1}(<p)} \partial_{i}^{\left(q_{1}\right)}\left\{\left(W_{i}^{(1) a}\left(\mathbf{r}_{q_{1}} ; p\right)+2 \sum_{q_{2}(<p) \neq q_{1}} W_{i}^{(2) a}\left(\mathbf{r}_{q_{1}} ; p, q_{2}\right)+\cdots, N\right),\right.
\end{aligned}
$$




$$
\begin{aligned}
& \left.\left.+(p-1) \sum_{q_{2}(<p) \neq q_{1}} \ldots \quad \sum_{q_{p-1}(<p) \neq q_{1}, \cdots, q_{p-2}} W_{i}^{(p-1) a}\left(\mathbf{r}_{p} ; p, q_{2}, \cdots, q_{p-1}\right)\right) T_{\left(q_{1}\right)}^{a}\right\} \Phi(1, \cdots, N ; t) \\
& -\frac{1}{2 m} \sum_{p=1}^{N} \sum_{q_{1}(<p)}\left\{\left(\sum_{q_{2}(<p) \neq q_{1}} W_{i}^{(1) a}\left(\mathbf{r}_{q_{1}} ; q_{2}\right)+\sum_{q_{2}(<p) \neq q_{1}} \sum_{q_{3}(<p) \neq q_{1}, q_{2}} W_{i}^{(2) a}\left(\mathbf{r}_{q_{1}} ; q_{2}, q_{3}\right)\right.\right. \\
& \left.+\cdots+\sum_{q_{2}(<p) \neq q_{1}} \cdots \sum_{q_{p-1}(<p) \neq q_{1}, \cdots, q_{p-2}} W_{i}^{(p-2) a}\left(\mathbf{r}_{p} ; q_{2}, \cdots, q_{p-1}\right)\right) T_{\left(q_{1}\right)}^{a} \\
& \cdot\left(W_{i}^{(1) b}\left(\mathbf{r}_{q_{1}} ; p\right)+2 \sum_{q_{2}^{\prime}(<p) \neq q_{1}} W_{i}^{(2) b}\left(\mathbf{r}_{q_{1}} ; p, q_{2}^{\prime}\right)+\cdots+(p-1) \sum_{q_{2}^{\prime}(<p) \neq q_{1}}\right. \\
& \left.\left.\cdots \sum_{q_{p-1}^{\prime}(<p) \neq q_{1}, q_{2}^{\prime}, \cdots, q_{p-2}^{\prime}} W_{i}^{(p-1) b}\left(\mathbf{r}_{q_{1}} ; p, q_{2}^{\prime}, \cdots, q_{p-1}^{\prime}\right)\right) T_{\left(q_{1}\right)}^{b}\right\} \Phi(1, \cdots, N ; t), \\
& C=-\frac{1}{2 m} \sum_{p=1}^{N} \sum_{q_{1}(<p)}\left\{\left(W_{i}^{(1) b}\left(\mathbf{r}_{q_{1}} ; p\right)+2 \sum_{q_{2}(<p) \neq q_{1}} W_{i}^{(2) b}\left(\mathbf{r}_{q_{1}} ; p, q_{2}\right)+\cdots\right.\right. \\
& \left.\left.+(p-1) \sum_{q_{2}(<p) \neq q_{1}} \ldots \sum_{q_{p-1}(<p) \neq q_{1}, \cdots, q_{p-2}} W_{i}^{(p-1) b}\left(\mathbf{r}_{q_{1}} ; p, q_{2}, \cdots, q_{p-1}\right)\right) T_{\left(q_{1}\right)}^{b}\right\} \partial_{i}^{\left(q_{1}\right)} \Phi(1, \cdots, N ; t) \\
& -\frac{1}{2 m} \sum_{p=1}^{N} \sum_{q_{1}(<p)}\left\{\left(W_{i}^{(1) b}\left(\mathbf{r}_{q_{1}} ; p\right)+2 \sum_{q_{2}(<p) \neq q_{1}} W_{i}^{(2) b}\left(\mathbf{r}_{q_{1}} ; p, q_{2}\right)+\cdots\right.\right. \\
& \left.+(p-1) \sum_{q_{2}(<p) \neq q_{1}} \cdots \sum_{q_{p-1}(<p) \neq q_{1}, \cdots, q_{p-2}} W_{i}^{(p-1) b}\left(\mathbf{r}_{q_{1}} ; p, q_{2}, \cdots, q_{p-1}\right)\right) T_{\left(q_{1}\right)}^{b} \\
& \cdot\left(\sum_{q_{2}^{\prime}(<p) \neq q_{1}} W_{i}^{(1) a}\left(\mathbf{r}_{q_{1}} ; q_{2}^{\prime}\right)+\sum_{q_{2}^{\prime}(<p) \neq q_{1}} \sum_{q_{3}^{\prime}(<p) \neq q_{1}, q_{2}^{\prime}} W_{i}^{(2) a}\left(\mathbf{r}_{q_{1}} ; q_{2}^{\prime}, q_{3}^{\prime}\right)+\cdots\right. \\
& \left.\left.+\sum_{q_{2}^{\prime}(<p) \neq q_{1}} \cdots \sum_{q_{p-1}^{\prime}(<p) \neq q_{1}, q_{2}^{\prime}, \cdots, q_{p-2}^{\prime}} W_{i}^{(p-2) a}\left(\mathbf{r}_{q_{1}} ; q_{2}^{\prime}, \cdots, q_{p-1}^{\prime}\right)\right) T_{\left(q_{1}\right)}^{a}\right\} \Phi(1, \cdots, N ; t), \\
& D=-\frac{1}{2 m} \sum_{p=1}^{N} \sum_{q_{1}(<p)}\left\{\left(W_{i}^{(1) b}\left(\mathbf{r}_{q_{1}} ; p\right)+2 \sum_{q_{2}(<p) \neq q_{1}} W_{i}^{(2) b}\left(\mathbf{r}_{q_{1}} ; p, q_{2}\right)+\cdots\right.\right. \\
& \left.\left.+(p-1) \sum_{q_{2}(<p) \neq q_{1}} \ldots \sum_{q_{p-1}(<p) \neq q_{1}, \cdots, q_{p-2}} W_{i}^{(p-1) b}\left(\mathbf{r}_{q_{1}} ; p, q_{2}, \cdots, q_{p-1}\right)\right) T_{\left(q_{1}\right)}^{b}\right\}^{2} \Phi(1, \cdots, N ; t) .
\end{aligned}
$$


We have here used the abbreviation $\Phi(1, \cdots, N ; t)$ for the N-body wave function.

Despite complicated-looking forms, the sum of the above four contributions conspires to produce a remarkably simple expression:

$$
\begin{aligned}
A+ & B+C+D= \\
& -\frac{1}{2 m} \sum_{p=1}^{N}\left\{\partial_{i}^{(p)}+\left(\sum_{q_{1} \neq p} W_{i}^{(1) a}\left(\mathbf{r}_{p} ; q_{1}\right)+\sum_{q_{1} \neq p} \sum_{q_{2} \neq p, q_{1}} W_{i}^{(2) a}\left(\mathbf{r}_{p} ; q_{1}, q_{2}\right)+\cdots\right.\right. \\
& \left.\left.+\sum_{q_{1} \neq p} \cdots \sum_{q_{p-1} \neq p, q_{1}, \cdots, q_{p-1}} W_{i}^{(N-1)}\left(\mathbf{r}_{p} ; q_{1}, \cdots, q_{N-1}\right)\right) T_{(p)}^{a}\right\}^{2} \Phi(1, \cdots, N ; t) .
\end{aligned}
$$

To see that, it is useful to recast the expression in the right hand side of Eq. (23) using the relation

$$
\begin{gathered}
\left(\sum_{q_{1} \neq p} W_{i}^{(1) a}\left(\mathbf{r}_{p} ; q_{1}\right)+\cdots+\sum_{q_{1} \neq p} \cdots \sum_{q_{p-1} \neq p, q_{1}, \cdots, q_{p-1}} W_{i}^{(N-1) a}\left(\mathbf{r}_{p} ; q_{1}, \cdots, q_{N-1}\right)\right) \\
=\sum_{q_{1}(<p)}\left(W_{i}^{(1) a}\left(\mathbf{r}_{p} ; q_{1}\right)+\sum_{q_{2}(<p) \neq q_{1}} W_{i}^{(2) a}\left(\mathbf{r}_{p} ; q_{1}, q_{2}\right)+\cdots\right. \\
\left.\quad+\sum_{q_{2}(<p) \neq q_{1}} \ldots \sum_{q_{p-1}(<p) \neq q_{1}, \cdots, q_{p-2}} W_{i}^{(p-1) a}\left(\mathbf{r}_{p} ; q_{1}, \cdots, q_{p-1}\right)\right) \\
+\sum_{s(>p)}\left(\begin{array}{l}
W_{i}^{(1) a}\left(\mathbf{r}_{p} ; s\right)+2 \sum_{q_{2}(<s) \neq p} W_{i}^{(2) a}\left(\mathbf{r}_{p} ; s, q_{2}\right)+\cdots \\
\left.+(s-1) \sum_{q_{2}(<s) \neq p} \sum_{q_{s-1}(<s) \neq p, q_{2}, \cdots, q_{s-2}} W_{i}^{(s-1) a}\left(\mathbf{r}_{p} ; s, q_{2}, \cdots, q_{s-1}\right)\right) .
\end{array}\right.
\end{gathered}
$$

At the same time we write the summation $\sum_{p=1}^{N} \sum_{q_{1}(<p)}$ appearing in $B, C$ and $D$ above as $\sum_{q_{1}=1}^{N} \sum_{p\left(>q_{1}\right)}$ and then rename the indices $\left(q_{1}, p\right)$ by $(p, s)$; the result will be the expressions for $B, C$ and $D$, all of which start with the summation $\sum_{p=1}^{N} \sum_{s(>p)}$. Then the remaining step is just to make sure that those expressions indeed match various contributions entering the right hand side of Eq. (23) when the relation (24) is used. Based on this observation and our earlier identification (9), we thus have the $N$-body 
Schrödinger equation in the desired form

$$
i \frac{\partial}{\partial t} \Phi(1, \cdots, N ; t)=-\frac{1}{2 m} \sum_{p=1}^{N}\left\{\partial_{i}^{(p)}+\mathcal{A}_{i}^{a}\left(\mathbf{r}_{p} ; 1, \cdots, p-1, p+1, \cdots, N\right) T_{(p)}^{a}\right\}^{2} \Phi(1, \cdots, N ; t) .
$$

This is the equivalent first-quantized description we have been after.

\section{DISCUSSIONS}

We have shown explicitly that the nonrelativistic quantum field theory defined by the Hamiltonian (2) with the operator constraints (3) is equivalent to that defined by the many-body Schrödinger equation (25) with the c-number constraints (10). Note that this complete equivalence between the two descriptions is not entirely an expected result, considering the nonlinear nature of our constraint equations.

The above $N$-body Schrödinger equation in Eq. (25) allows an immediate physical interpretation: each particle is under the influence of a non-Abelian vector potential produced by other particles (which act as non-Abelian point sources in the sense of Eq. (10)). Especially, for $N=2$, we know the exact expression for the vector potential in the Coulomb gauge ( see Eq. (8)),

$$
\begin{aligned}
\mathcal{A}_{i}^{a}\left(\mathbf{r}_{1} ; 2\right) & =W_{i}^{(1) a}(\mathbf{r} ; 2) \\
& =\frac{i}{2 \pi \kappa} \epsilon^{i j} \frac{\left(x_{1}-x_{2}\right)_{j}}{\left|\mathbf{r}_{1}-\mathbf{r}_{2}\right|} \\
& =-\mathcal{A}_{i}^{a}\left(\mathbf{r}_{2} ; 1\right)
\end{aligned}
$$

Using complex coordinates $z_{p}=x_{p}+i y_{p}$ and $\overline{z_{p}}=x_{p}-i y_{p}(p=1,2)$, the 2-body Schrödinger equation can then be conveniently written as

$$
i \frac{\partial}{\partial t} \Phi(1,2 ; t)=-\frac{1}{m} \sum_{p=1,2}\left(\overline{\mathcal{D}}_{(p)} \mathcal{D}_{(p)}+\mathcal{D}_{(p)} \overline{\mathcal{D}}_{(p)}\right) \Phi(1,2 ; t)
$$

where

$$
\begin{array}{ll}
\mathcal{D}_{(1)}=\frac{\partial}{\partial z_{1}}-\frac{1}{4 \pi \kappa} \frac{T_{(1)}^{a} T_{(2)}^{a}}{z_{1}-z_{2}}, & \overline{\mathcal{D}}_{(1)}=\frac{\partial}{\partial \bar{z}_{1}}+\frac{1}{4 \pi \kappa} \frac{T_{(1)}^{a} T_{(2)}^{a}}{\bar{z}_{1}-\bar{z}_{2}} \\
\mathcal{D}_{(2)}=\frac{\partial}{\partial z_{2}}-\frac{1}{4 \pi \kappa} \frac{T_{(1)}^{a} T_{(2)}^{a}}{z_{2}-z_{1}}, & \overline{\mathcal{D}}_{(2)}=\frac{\partial}{\partial \bar{z}_{2}}+\frac{1}{4 \pi \kappa} \frac{T_{(1)}^{a} T_{(2)}^{a}}{\bar{z}_{2}-\bar{z}_{1}}
\end{array}
$$


Introducing a new 2-body wave function $\Psi(1,2 ; t)$ by

$$
\Phi(1,2 ; t)=\exp \left\{-\frac{1}{4 \pi \kappa}\left(\ln \left(z_{1}-z_{2}\right)\left(\bar{z}_{1}-\bar{z}_{2}\right)\right) T_{(1)}^{a} T_{(2)}^{a}\right\} \Psi(1,2 ; t)
$$

we can recast Eq. (27) as

$$
i \frac{\partial}{\partial t} \Psi(1,2 ; t)=-\frac{1}{m} \sum_{p=1,2}\left(\bar{\nabla}_{(p)} \nabla_{(p)}+\nabla_{(p)} \bar{\nabla}_{(p)}\right) \Psi(1,2 ; t),
$$

where $\bar{\nabla}_{(1)}=\frac{\partial}{\partial \bar{z}_{1}}, \quad \bar{\nabla}_{(2)}=\frac{\partial}{\partial \bar{z}_{2}}, \quad \nabla_{(1)}=\frac{\partial}{\partial z_{1}}-\frac{1}{2 \pi \kappa} \frac{T_{(1)}^{a} T_{(2)}^{a}}{z_{1}-z_{2}}$, and $\nabla_{(2)}=\frac{\partial}{\partial z_{2}}-\frac{1}{2 \pi \kappa} \frac{T_{(1)}^{a} T_{(2)}^{a}}{z_{2}-z_{1}}$. This is precisely what one has in the holomorphic gauge [6, 7]. But, Eq. (29) being not a unitary transformation, the normalization for $\Psi$ will be unconventional while $\Phi$ satisfies the conventional normalization. We also note that the 2-body scattering based on Eq. (27) is not much different from the Abelian Aharonov-Böhm scattering [6, 7, 9]. But, with three or more particles, we do not even have the explicit expressions for $\mathcal{A}_{i}^{a}$ (in the Coulomb gauge) and a full quantum treatment for this case remains as a nontrivial future problem. Possibly one can look for perturbative solutions to Eq. (7) (treating $\frac{1}{\kappa}$ as an expansion parameter) to find $W_{i}^{(n) a}$ for $n \geq 2$, although an exact treatment should be much more desirable.

Note: There appeared a very recent paper by Bak, Jackiw, and Pi in which a systematic axial gauge treatment for the same model has been given [10]. 


\section{Acknowledgements}

We would like to thank R. Jackiw, S.-Y. Pi, D. Bak, T. Lee and P. Oh for comments

and useful discussions. This work was supported in part by the Korea Science and Engineering Foundation through the Center for Theoretical Physics, SNU and also by the Ministry of Education, Korea.

\section{References}

[1] R. Jackiw and S.-Y. Pi, Phys. Rev. D 42, 3500 (1990).

[2] M. Leinaas and J. Myrheim, Nuovo Cimento B 37, 1 (1977); F. Wilczek, Phys. Rev. Lett. 48, 1144, (1982); Y. S. Wu, Phys. Rev. Lett. 52, 2103 (1984).

[3] J. Fröhlich, in Nonperturbative Quantum Field Theory, edited by G. 't Hooft et al. (New York, Plenum, 1988).

[4] J. Schonfeld, Nucl. Phys. B 185, 157 (1981); R. Jackiw and S. Templeton, Phys. Rev. D 23, 2291 (1981); S. Deser, R. Jackiw, and S. Templeton, Phys. Rev. Lett. 48, 975 (1982); C. Hagen, Ann. Phys. (N.Y.) 157, 342 (1984).

[5] S. Deser, R. Jackiw, and S. Templeton, Ann. Phys. (N.Y.) 140, 372 (1982).

[6] E. Verlinde, in Modern Quantum Field Theory, edited by S. Das et al (World Scientific, Singapore, 1991).

[7] T. Lee and P. Oh, Ann. Phys. (N.Y.) (in press); Phys. Lett. B 319, 497 (1993).

[8] C.-L. Ho and Y. Hosotani, Int, J. Mod. Phys. A 7, 5797 (1992); C. Kim, C. Lee, P. Ko, B.-H. Lee and H. Min, Phys. Rev. D 48, 1821 (1993).

[9] F. Wilczek and Y. S. Wu, Phys. Rev. Lett. 65, 13 (1990).

[10] D. Bak, R. Jackiw, and S.-Y. Pi, "Non-Abelian Chern-Simons Particles and their Quantization", MIT preprint, CTP \# 2276 ( hep-th/9402057). 